\title{
Plasma Apelin, Visfatin and Resistin Levels in Patients with First Episode Psychosis and Chronic Schizophrenia
}

\author{
Musa Sahpolat ${ }^{1}$, Mustafa $\mathrm{Ari}^{2}$, Mehmet Hanifi Kokacya ${ }^{2}$ \\ ${ }^{1}$ Department of Psychiatry, Kilis State Hospital, Kilis, ${ }^{2}$ Deparment of Psychiatry, Faculty of Medicine, Mustafa Kemal University, Hatay, Turkey
}

\begin{abstract}
Objective: This study aims to investigate the possible relationship between plasma concentrations of apelin, visfatin and resistin levels of first episode psychosis patients and chronic schizophrenia patients.

Methods: A total number of 29 untreated patients with first episode psychosis, 30 chronic schizophrenia and 29 randomly selected weight- and body mass index-matched healthy volunteers were included. The Diagnostic and Statistical Manual of Mental Disorders 4th edition, Positive and Negative Syndrome Scale (PANSS) and Clinical Global Impression Scale were applied to the patient groups. The enzyme-linked immunosorbent assay method was used to measure plasma apelin, visfatin and resistin levels.

Results: There was no difference in age, marital status, occupation, and BMI between the groups. Plasma apelin levels were significantly higher in first episode psychosis group than chronic schizophrenia and control group. There was no statistically significant difference in plasma visfatin levels between the groups: first episode psychosis group, chronic schizophrenia and control group. Plasma resistin levels were higher in both first episode psychosis group and chronic schizophrenia group than the control group. There was no statistically significant correlation between plasma apelin and resistin levels and total PANSS scores in the group of patients.

Conclusion: To our knowledge, this study is the first which investigates the plasma apelin, visfatin and resistin levels in patients with first episode psychosis and chronic schizophrenia. Based on the results of this study, apelin and resistin may be related with some central nervous system pathologies, including the severity of a psychiatric disorder.
\end{abstract}

KEY WORDS: First episode psychosis; Schizophrenia; Apelin; Nicotinamide phosphoribosyltransferase; Resistin.

\section{INTRODUCTION}

Schizophrenia is a complex disease and a devastating neuropsychiatric illness affecting approximately $1 \%$ of the total population worldwide over the average lifetime $[1,2]$. Its etiology is unclear yet. One of the important hypotheses to explain its pathogenesis is about immune dysfunction $[1,2]$. One of the most interesting and challenging areas of research is the role of adipocytes in the etiology of mental disorders [2,3]. There are numerous descriptions of an association between chronic inflammation and adipokines of the central nervous system (CNS),

\footnotetext{
Received: April 16, 2019/Revised: June 21, 2019

Accepted: July 23, 2019

Address for correspondence: Musa Sahpolat

Department of Psychiatry, Kilis State Hospital, Kilis 79100, Turkey

E-mail: drmsahpolat@hotmail.com

ORCID: https://orcid.org/0000-0002-0022-2389
}

and schizophrenia [2-4]. Several types of adipocyte receptor have been found in several central nervous system areas, and have been shown to affect brain function through neuroplastic processes [4-6]. These findings clearly showed the immune theory of psychiatric diseases such as schizophrenia $[1,3,6,7]$.

Adipose tissue, it secretes a protein called adipokine, which is known to play roles in several physiological processes in the body such as eating, appetite, energy balance, insulin and glucose metabolism, lipid metabolism, regulation of blood pressure, vascular remodeling, coagulation, inflammation [8]. Some secretions of this tissue are interleukin (IL)-6, tumor necrosis factor (TNF)- $\alpha$, insulin-like growth factor-1, C-reactive protein, sex hormones, adiponectin, resistin, apelin and visfatin [9].

Apelin is a relatively newly discovered neuropeptide that is the endogenous ligand for the G-protein-coupled (APJ) receptor, and it impacts by binding to APJ [10].

(c) This is an Open-Access article distributed under the terms of the Creative Commons Attribution Non-Commercial License (http://creativecommons.org/licenses/by-nc/4.0) which permits unrestricted non-commercial use, distribution, and reproduction in any medium, provided the original work is properly cited. 
Apelin is expressed in both brain and peripheral tissues in human. In the brain, apelin is expressed in thalamus, frontal cortex and hippocampus, while it is also expressed in placenta, heart, lung and other peripheral tissues. The apelin receptors and apelin are widely distributed in the CNS $[10,11]$ suggesting that apelin may be of importance in the regulation of certain CNS functions. Some studies have shown that apelin plays role in cardiovascular function, anterior pituitary function, regulation of certain CNS functions and the regulation of fluid homeostasis; also takes part in the suppression of apoptosis and acts as a co-receptor in human immunodeficiency virus (HIV) infection $[10,11]$.

Visfatin has been recently identified as a peptide predominantly expressed in and secreted from visceral fat in both humans and mice. It is stated that plasma visfatin levels are associated with visceral fat mass, Type 2 diabetes mellitus and metabolic syndrome in humans [12]. Visfatin was originally identified as a pre-B cell colony enhancing factor and is thought to play roles in immune response and inflammation. Moreover, human recombinant visfatin treatment increases the secretion of inflammatory cytokines IL-1, IL-6, TNF- $\alpha$ [13]. Thus, there is some evidence to suggest that visfatin activates proinflammatory cytokines in human monocytes [14]. However, little is known about its function in the brain.

Resistin is a recently identified proinflammatory adipocytokine. Human resistin has also been detected in tissues like placenta, skeletal muscle, small intestine, spleen, stomach, thymus, thyroid gland, and uterus. However, resistin is predominantly expressed in macrophages in humans $[15,16]$. Recently, resistin has been found to be involved in inflammation and regulation of other cytokines as well. On the other hand, some proinflammatory cytokines can induce the expression of resistin $[15,16]$. One study reported that resistin effects closely mirror leptin-induced inhibition of dopamine and norepinephrine release in the hypothalamus, supporting a role for both catecholamines as central mediators of adipocyte-repleted signaling in the CNS [16].

It is known that inflammatory process plays a role in the etiology of schizophrenia. In this study, we aimed to investigate the possible relationship between first episode psychosis, chronic schizophrenia and plasma concentrations of apelin, visfatin and resistin in the inflammatory process.

\section{METHODS}

\section{Participants}

This study included a total of 29 untreated patients with first episode psychosis, 30 chronic schizophrenia and 29 randomly selected weight- and body mass index-matched healthy volunteers admitted to Mustafa Kemal University, Faculty of Medicine, Training and Research Hospital, Psychiatry outpatient clinic were included. Three groups were formed for the study. The first group consisted of patients with non-effective drug-naive first episode psychosis. Second group consisted of patients with chronic schizophrenia. And the third group was healthy control group. Diagnoses of the patients were made in accordance with the Diagnostic and Statistical Manual of Mental Disorders 4th edition schizophrenia diagnosis criteria. The patients with the first attack who take antipsychotics and patients with affective psychosis were excluded from the study. Individuals with any other chronic disease such as diabetes mellitus, hypertension, hyperlipidemia, or neurological disorders, who were under 18 years of age and those over 65 years of age and pregnant women in the menstrual cycle were excluded from the study. People with any additional psychiatric condition were excluded; also those with mental retardation and those with organic brain damage were not included in the study. For the control group, people with any psychiatric or medical condition were excluded from the study. Before enrollment, the relationship between plasma apelin, visfatin and resistin levels and socio-demographic characteristics was examined. A written informed consent was obtained from each participant. The study protocol was research ethics approval was obtained from the Ethics Committee of the Medical School of Mustafa Kemal University (no. 201335). The study was conducted in accordance with the principles of the Declaration of Helsinki.

Socio-demographic data, such as age, sex, educational level, marital status, occupational status, mental status, smoking, height, weight, and body mass index (BMI), was collected. The severity of psychosis symptoms in the patients was evaluated using the Positive and Negative Syndrome Scale (PANSS) [17]. Physical and neurological examinations were performed in all participants. 
Table 1. Sociodemographic characteristics of the groups

\begin{tabular}{lcccl}
\hline \multicolumn{1}{c}{ Variable } & $\begin{array}{c}\text { First episode psychosis } \\
\text { group }(\mathrm{n}=29)\end{array}$ & $\begin{array}{c}\text { Chronic schizophrenia } \\
\text { group }(\mathrm{n}=30)\end{array}$ & $\begin{array}{c}\text { Healthy control } \\
\text { group }(\mathrm{n}=29)\end{array}$ & Significance \\
\hline Age $(\mathrm{yr})$ & $31.14 \pm 12.12$ & $33.87 \pm 10.49$ & $30.21 \pm 8.13$ & $p=0.374^{\mathrm{a}}, \mathrm{F}=0.995$ \\
Sex $(\mathrm{female} / \mathrm{male})$ & $12 / 17$ & $13 / 17$ & $16 / 13$ & $p=0.521^{\mathrm{b}}, \chi^{2}=1.303$ \\
BMI $\left(\mathrm{kg} / \mathrm{m}^{2}\right)$ & $24.89 \pm 5.14$ & $26.59 \pm 4.71$ & $23.94 \pm 3.94$ & $p=0.089^{\mathrm{a}}, \mathrm{F}=2.491$ \\
Total PANSS & $101.90 \pm 16.35$ & $92.07 \pm 21.56$ & - & $p=0.046^{\mathrm{c}, *}, U=303.50$ \\
\hline
\end{tabular}

Values are presented as mean \pm standard deviation or number only.

BMI, body mass index; PANSS, Positive and Negative Syndrome Scale.

${ }^{\mathrm{a}}$ One-way ANOVA test, ${ }^{\mathrm{b}}$ Pearson's chi-square test, 'Mann - Whitney $U$ test; ${ }^{*} p<0.05$.

\section{Blood Collection and Clinical Laboratory Measurements}

From the patients' forearm, a venous blood sample was taken after 12 hours of fasting at 08:00 AM. Routine psychiatric examination, PANSS [18] and Clinical Global Impression [19] scale were applied on the blood collection day. All collected bloods were centrifuged for $15 \mathrm{mi}-$ nutes $(3,000 \times g)$ over two hours, and sera were stored at $-70^{\circ} \mathrm{C}$. After the blood collection, plasma apelin, visftain and resistin levels were measured by enzyme- linked immunosorbent assay (ELISA) method using the apelin, visfatin and resistin ELISA kit (Biovendor Laboratory Medicine Inc., Brno, CzechRepublic).

\section{Statistical Analysis}

Statistical analysis was performed using Number Cruncher Statistical System (NCSS) version 2007 software (NCSS, Kaysville, UT, USA). Descriptive data was expressed in mean, standard deviation, frequency, and rate. Student $t$ test for the two-group-comparison of quantitative data with normal distribution and Mann-Whitney $U$ test for the comparison of not-normally-distributed variables were used. For the comparison of three-or-more groups with normal distributions, One-way ANOVA and Bonferroni post-hoc test; for the comparison of three-ormore groups without normal distributions, Kruskal -Wallis test; and to detect the group causing the differences, Mann-Whitney $U$ test were used. For comparing the qualitative data Pearson's chi-square test, FisherFreeman-Halton test was used. Evaluation of the relationship between the variables was made by Pearson correlation analysis and Spearman's correlation analysis. $p$ values of $<0.05$ were considered statistically significant.

\section{RESULTS}

All groups had similar demographic characteristics. The demographic and biochemical characteristics were shown in Table 1.

Statistically significant differences were found between the groups for apelin levels ( $p=0.026 ; \chi^{2}=7.338$ ). According to pairwise comparisons; apelin levels of the first episode psychosis patients were significantly higher than the chronic schizophrenia and control group patients ( $p=0.034 ; p=0.012$, respectively). There was no significant difference in apelin levels between the chronic schizophrenia and control group patients $(p=0.644)$. There was no statistically significant difference in visfatin levels between the groups $(p=0.109 ; \mathrm{F}=2.272)$. In the comparison of resistin levels between the groups, a high level of statistically significant differences was found ( $p=$ 0.007; $\left.\chi^{2}=10.000\right)$. According to pairwise comparison; resistin levels of the first episode psychosis and chronic schizophrenia groups were significantly higher than the controls ( $p=0.003 ; p=0.016$, respectively). There was no statistically significant difference in resistin levels between the first episode psychosis and chronic schizophrenia patients $(p=0.490)$. It was shown in Table 2 .

There was no statistically significant difference in sociodemographic variables in terms of the mean plasma apelin, visfatin and resistin levels between three groups. Plasma apelin, visfatin and resistin levels of three groups were not statistically significantly associated with BMI and smoking $(p>0.05)$. There was no statistically significant relationship between the Total PANSS score and plasma apelin, visfatin, resistin levels in patients with first episode psychosis and chronic schizophrenia $(p>0.05)$. 
Table 2. Plasma apelin, visfatin and resistin evaluations for groups

\begin{tabular}{|c|c|c|c|c|c|}
\hline Variable & $\begin{array}{l}\text { First episode psychosis } \\
\text { group }(\mathrm{n}=29)\end{array}$ & $\begin{array}{l}\text { Chronic schizophrenia } \\
\quad \text { group }(n=30)\end{array}$ & $\begin{array}{l}\text { Healthy control } \\
\text { group }(n=29)\end{array}$ & Significance & $\begin{array}{l}\text { Pairwise } \\
\text { comparisons }^{\mathrm{c}, \mathrm{d}} \\
\text { ( } p \text { value })\end{array}$ \\
\hline Apelin & & & & $p=0.026^{\mathrm{b}_{*},}$ & $0.034^{*}$ \\
\hline Min-Max (median) & $26.1-311.3(147.9)$ & $10.0-236.9(107.9)$ & $32.0-260.8(93.3)$ & $\chi^{2}=7.338$ & $0.012^{*}$ \\
\hline Mean \pm standard deviation & $156.88 \pm 86.09$ & $108.04 \pm 59.47$ & $104.01 \pm 56.60$ & & 0.644 \\
\hline Visfatin & & & & $p=0.109^{\mathrm{a}}$ & 0.361 \\
\hline Min-Max (median) & $3.2-7.6(5.9)$ & $4.4-11.3(6.0)$ & $4.8-8.2(6.4)$ & $\mathrm{F}=2.272$ & 0.134 \\
\hline Mean \pm standard deviation & $5.78 \pm 1.10$ & $6.24 \pm 1.35$ & $6.38 \pm 0.86$ & & 1.000 \\
\hline Resistin & & & & $p=0.007^{\mathrm{b}, * *}$, & 0.490 \\
\hline Min-Max (median) & $10.7-16(14.9)$ & $9.7-16(14.1)$ & $9.1-15.9(13.2)$ & $\chi^{2}=10.000$ & $0.003^{* *}$ \\
\hline Mean \pm standard deviation & $14.19 \pm 1.44$ & $13.89 \pm 1.62$ & $12.79 \pm 1.80$ & & $0.016^{*}$ \\
\hline
\end{tabular}

${ }^{a}$ One-way ANOVA test, ${ }^{b}$ Kruskal -Wallis test, ${ }^{\mathrm{C}}$ Mann - Whitney $U$ test, ${ }^{\mathrm{d}}$ Adjustment for Multiple Comparisons: Bonferroni post-hoc test; ${ }^{*} p<$ $0.05,{ }^{* *} p<0.01$.

\section{DISCUSSION}

There is limited information about the role of apelin, visfatin, and resistin in psychiatric disorders such as schizophrenia in the inflammatory process. This study was designed to determine whether plasma levels of apelin, visfatin, and resistin were altered in schizophrenia without additional comorbid diseases. To eradicate the possible effect of obesity on the apelin, visfatin and resistin levels, plasma concentrations of apelin, visfatin and resistin were corrected with the BMI.

Dysregulation of immune system has a significant place in the proofs related to etiopathogenesis of schizophrenia. It is thought to be there is a close relation between schizophrenia and autoimmune processes. There are strong data particularly on proinflammatory processes in schizophrenia $[1,3]$. Findings of our study are also supportive of this hypothesis.

One of the most substantial finding in our study is that we found plasma apelin levels of first episode psychosis patients were significantly higher than the chronic schizophrenic group and control groups. There are studies about the emotional consequences of apelin administration to the CNS and studies investigating the relationship between plasma apelin levels and some psychiatric disorders; but no human study focusing on the apelin levels in patients with chronic schizophrenia and the first episode psychosis was available. Apelin was reported to be in many areas of the brain, such as cortex, hypothalamus, hippocampus, pituitary, spinal cord, cerebellum, corpus callosum, substantia nigra, the dorsal raphe nucleus, the central gray matter, amygdala, subthalamic nucleus, caudate nucleus, and especially in supraoptic and paraventricular nuclei [19-21]. These areas are known to be related to emotions and being released from these areas suggests a possible role of apelin in emotional behavior. Suppression of eating behavior with apelin-13 $[22,23]$ and the close relationship between eating behavior and emotional behavior $[24,25]$ support this opinion. For example, the anorexigenic CRF and $\alpha-M S H$ showed an anxiety and depression-like effect $[26,27]$. The orexigenic Neuropeptide $Y$ and ghrelin reduces the anxiety-depression dependent behavior [28,29]. Apelin has been demonstrated to exert neuroprotective action under a number of experimental conditions [21,30]; in culture, it markedly prevents apoptosis in mouse cortical neurons [31] and it protects hippocampal neurons against $\mathrm{N}$-Methyl-D-aspartate excitotoxity [32]. No data are as yet available regarding its action on psychosis.

One of the most substantial finding in our study is that plasma visfatin levels were not statistically difference between the three groups. In contrast to other studies in literature, our finding suggests that visfatin has no effect on both acute and chronic phases of psychosis. Visfatin, also known as pre-B-cell colony-enhancing factor, plasma levels correlated with inflammation cytokines or mediator. Visfatin has been found to be also expressed in animal and human brain $[33,34]$. Moreover, higher plasma visfatin levels are associated with ischemic stroke [35], and visfatin has a neuroprotective effect in ischemia through its enzymatic activity for nicotinamide adenine dinucleotide production that can ameliorate mitochondrial dys- 
function [36]. One study demonstrated that increasing levels of TNF- $\alpha$ in the serum of children with autism spectrum disorders and also observed a significant positive correlation between TNF- $\alpha$ and resistin and visfatin [37]. Some studies suggest that there was no significant association between the concentration of circulating visfatin and presence of eating disorders [38,39]. At present, there are no sufficient studies to explain the relationship between visfatin and psychosis.

The most substantial finding in our study is that we found high plasma resistin levels in both first episode psychosis and chronic schizophrenia patients. This, in line with the literature, suggests a role of resistin in inflammatuar process in both acute and chronic phase of psychosis. According to our findings, resistin may be a component of inflammatuar process both acute and chronic stress. In humans, resistin appears to be an inflammatory molecule primarily expressed in monocytic cells, from which it is secreted. The correlation between resistin with inflammatory markers (e.g., IL-6, TNF- $\alpha$ ) is especially considerable given the observation that resistin is produced by macrophages in response to inflammatory cytokines $[7,40]$. Resistin has been implicated in the pathogenesis of several inflammatory central nervous system disorders [40]. Recently studies suggested that there is an association between inflammatory agents produced by adipose tissue and risk of depression [7]. Some studies have reported a positive correlation between resistin levels in the blood and atypical, melancholic subtypes of major depressive disorders $[41,42]$. This association may be related to the reduction in intrasynaptic concentration of monoamines by resistin via inhibition of release of norepinephrine and dopamine in the hypothalamus [43]. It has been suggested that resistin is involved in the pathogenesis of bipolar disorder. A recent study reported increased levels of resistin in patients with bipolar disorder, the specific role of resistin in the pathogenesis of the illness is still unknown [44]. Some studies reported reduced concentration of resistin have been observed in patients with anorexia nervosa [45], autism spectrum disorders [46] and obsessive compulsive disorder [47]. In a study by Balotsev et al. [48] was found ferritin and resistin levels of drug naive first episode psychosis patients were significantly higher than the chronic schizophrenic patients. It was shown that after seven months of antipsychotic drug treatment in first episode psychosis patients the strongest decline was established for ferritin, followed by resistin. This study supports our findings on relation between resistin and psychosis.

Nonetheless, there are some limitations to this study. This is a cross-sectional study with a small sample size. There is not sufficient data about the first episode psychosis and chronic schizophrenia as a study which compares these molecules (apelin, visfatin, and resistin) have not been done. Levels of other inflammatory markers (e.g., ferritin, IL-6, TNF- $\alpha$ ) could be examined, together with apelin, visfatin and resistin.

In conclusion, to our knowledge, this study is the first which investigates the plasma apelin, visfatin and resistin levels in patients with first episode psychosis. Based on the results of this study, apelin and resistin may be related with some central nervous system pathologies, including the severity of a psychiatric disorder. However, further large-scale studies are required to establish a conclusion.

\section{- Acknowledgments}

The authors would like to thank the participants.

\section{- Conflicts of Interest}

No potential conflict of interest relevant to this article was reported.

\section{Author Contributions}

Conceptualization: Musa Sahpolat, Mustafa Ari, Mehmet Hanifi Kokacya. Data acquisition: Musa Sahpolat. Formal analysis: Musa Sahpolat, Mustafa Ari, Mehmet Hanifi Kokacya. Funding: Musa Sahpolat, Mustafa Ari, Mehmet Hanifi Kokacya. Supervision: Musa Sahpolat, Mustafa Ari, Mehmet Hanifi Kokacya. Writingoriginal draft: Musa Sahpolat. Writing-review \& editing: Musa Sahpolat, Mustafa Ari, Mehmet Hanifi Kokacya.

\section{ORCID}

Musa Sahpolat

https://orcid.org/0000-0002-0022-2389

Mustafa Ari https://orcid.org/0000-0002-8003-1661 Mehmet Hanifi Kokacya

https://orcid.org/0000-0002-2961-7229

\section{REFERENCES}

1. Bolu A, Aydın MS, Akgün A, Coşkun A, Garip B, Öznur T, et al. Serum levels of high sensitivity C-reactive protein in drug-naïve first-episode psychosis and acute exacerbation of 
schizophrenia. Clin Psychopharmacol Neurosci 2019;17: 244-249.

2. Beumer W, Drexhage RC, De Wit H, Versnel MA, Drexhage $\mathrm{HA}$, Cohen D. Increased level of serum cytokines, chemokines and adipokines in patients with schizophrenia is associated with disease and metabolic syndrome. Psychoneuroendocrinology 2012;37:1901-1911.

3. Strous RD, Shoenfeld Y. Schizophrenia, autoimmunity and immune system dysregulation: a comprehensive model updated and revisited. J Autoimmun 2006;27:71-80.

4. Nillni EA, Vaslet C, Harris M, Hollenberg A, Bjørbak C, Flier JS. Leptin regulates prothyrotropin-releasing hormone biosynthesis. Evidence for direct and indirect pathways. I Biol Chem 2000;275:36124-36133.

5. Rybakowski F, Slopien A, Tyszkiewicz-Nwafor M. Inverse relationship between leptin increase and improvement in depressive symptoms in anorexia nervosa. Neuro Endocrinol Lett 2014;35:64-67.

6. Beumer W, Gibney SM, Drexhage RC, Pont-Lezica L, Doorduin J, Klein HC, et al. The immune theory of psychiatric diseases: a key role for activated microglia and circulating monocytes. J Leukoc Biol 2012;92:959-975.

7. Shelton RC, Miller AH. Inflammation in depression: is adiposity a cause? Dialogues Clin Neurosci 2011;13:41-53.

8. Antoniades C, Antonopoulos AS, Tousoulis D, Stefanadis C. Adiponectin: from obesity to cardiovascular disease. Obes Rev 2009;10:269-279.

9. Ronti T, Lupattelli G, Mannarino $\mathrm{E}$. The endocrine function of adipose tissue: an update. Clin Endocrinol (Oxf) 2006;64: 355-365.

10. Tatemoto K, Hosoya M, Habata Y, Fujii R, Kakegawa T, Zou $\mathrm{MX}$, et al. Isolation and characterization of a novel endogenous peptide ligand for the human APJ receptor. Biochem Biophys Res Commun 1998;251:471-476.

11. Reaux A, De Mota N, Skultetyova I, Lenkei Z, El Messari S, Gallatz K, et al. Physiological role of a novel neuropeptide, apelin, and its receptor in the rat brain. I Neurochem 2001; 77:1085-1096.

12. Filippatos TD, Derdemezis CS, Gazi IF, Lagos K, Kiortsis DN, Tselepis AD, et al. Increased plasma visfatin levels in subjects with the metabolic syndrome. Eur I Clin Invest 2008;38: 71-72.

13. Ognjanovic S, Bryant-Greenwood GD. Pre-B-cell colony-enhancing factor, a novel cytokine of human fetal membranes. Am J Obstet Gynecol 2002;187:1051-1058.

14. Moschen AR, Kaser A, Enrich B, Mosheimer B, Theurl M, Niederegger $\mathrm{H}$, et al. Visfatin, an adipocytokine with proinflammatory and immunomodulating properties. I Immunol 2007; 178:1748-1758.

15. Demirci S, Aynalı A, Demirci K, Demirci S, Arıdoğan BC. The serum levels of resistin and its relationship with other proinflammatory cytokines in patients with Alzheimer's disease. Clin Psychopharmacol Neurosci 2017;15:59-63.
16. Brunetti L, Michelotto B, Orlando G, Vacca M. Leptin inhibits norepinephrine and dopamine release from rat hypothalamic neuronal endings. Eur J Pharmacol 1999;372:237-240.

17. Kay SR, Fiszbein A, Opler LA. The positive and negative syndrome scale (PANSS) for schizophrenia. Schizophr Bull 1987;13:261-276.

18. Guy W. ECDEU assessment manual for psychopharmacology. Rockville:US Department of Health and Human Services Publication; 1976. p.218-222.

19. O'Carroll AM, Lolait SJ, Harris LE, Pope GR. The apelin receptor APJ: journey from an orphan to a multifaceted regulator of homeostasis. J Endocrinol 2013;219:R13-R35.

20. Kleinz MJ, Davenport AP. Emerging roles of apelin in biology and medicine. Pharmacol Ther 2005;107:198-211.

21. Cheng B, Chen J, Bai B, Xin Q. Neuroprotection of apelin and its signaling pathway. Peptides 2012;37:171-173.

22. Lv SY, Yang YJ, Qin YJ, Mo JR, Wang NB, Wang YJ, et al. Central apelin-13 inhibits food intake via the CRF receptor in mice. Peptides 2012;33:132-138.

23. Sunter D, Hewson AK, Dickson SL. Intracerebroventricular injection of apelin-13 reduces food intake in the rat. Neurosci Lett 2003;353:1-4.

24. Christensen L. The effect of food intake on mood. Clin Nutr 2001;20:161-166.

25. Licinio-Paixao J. Hyperinsulinemia; a mediator of decreased food intake and weight loss in anorexia nervosa and major depression. Med Hypotheses 1989;28:125-130.

26. Kokare DM, Dandekar MP, Singru PS, Gupta GL, Subhedar NK. Involvement of alpha-MSH in the social isolation induced anxiety- and depression-like behaviors in rat. Neuropharmacology 2010;58:1009-1018.

27. Holsboer $\mathrm{F}$. The rationale for corticotropin-releasing hormone receptor (CRH-R) antagonists to treat depression and anxiety. J Psychiatr Res 1999;33:181-214.

28. Stogner KA, Holmes PV. Neuropeptide-Y exerts antidepressant-like effects in the forced swim test in rats. Eur J Pharmacol 2000;387:R9-R10.

29. Lutter M, Sakata I, Osborne-Lawrence S, Rovinsky SA, Anderson JG, Jung S, et al. The orexigenic hormone ghrelin defends against depressive symptoms of chronic stress. Nat Neurosci 2008; 11:752-753.

30. Newson MJF, Roberts EM, Pope GR, Lolait SJ, O'Carroll AM. The effects of apelin on hypothalamic-pituitary-adrenal axis neuroendocrine function are mediated through corticotrophin-releasing factor- and vasopressin-dependent mechanisms. J Endocrinol 2009;202:123-129.

31. Zeng XJ, Yu SP, Zhang L, Wei L. Neuroprotective effect of the endogenous neural peptide apelin in cultured mouse cortical neurons. Exp Cell Res 2010;316:1773-1783.

32. O’Donnell LA, Agrawal A, Sabnekar P, Dichter MA, Lynch DR, Kolson DL. Apelin, an endogenous neuronal peptide, protects hippocampal neurons against excitotoxic injury. I Neurochem 2007;102:1905-1917. 
33. Kitani T, Okuno S, Fujisawa H. Growth phase-dependent changes in the subcellular localization of pre-B-cell colony-enhancing factor. FEBS Lett 2003;544:74-78.

34. Samal B, Sun Y, Stearns G, Xie C, Suggs S, McNiece I. Cloning and characterization of the $C D N A$ encoding a novel human pre-B-cell colony-enhancing factor. Mol Cell Biol 1994;14: 1431-1437.

35. Lu LF, Yang SS, Wang CP, Hung WC, Yu TH, Chiu CA, et al. Elevated visfatin/pre-B-cell colony-enhancing factor plasma concentration in ischemic stroke. I Stroke Cerebrovasc Dis 2009; 18:354-359.

36. Zhang W, Xie Y, Wang T, Bi J, Li H, Zhang LQ, et al. Neuronal protective role of PBEF in a mouse model of cerebral ischemia. J Cereb Blood Flow Metab 2010;30:1962-1971.

37. Ghaffari MA, Mousavinejad E, Riahi F, Mousavinejad M, Afsharmanesh MR. Increased serum levels of tumor necrosis factor-alpha, resistin, and visfatin in the children with autism spectrum disorders: a case-control study. Neurol Res Int 2016;:2016:9060751.

38. Dostálová I, Sedlácková D, Papezová H, Nedvídková J, Haluzík M. Serum visfatin levels in patients with anorexia nervosa and bulimia nervosa. Physiol Res 2009;58:903-907.

39. Ziora K, Oświęcimska J, Swiętochowska E, Ziora D, Stojewska $\mathrm{M}$, Suwała A, et al. Assessment of serum visfatin levels in girls with anorexia nervosa. Clin Endocrinol (Oxf) 2012;76:514519.

40. Patel L, Buckels AC, Kinghorn IJ, Murdock PR, Holbrook JD, Plumpton C, et al. Resistin is expressed in human macrophages and directly regulated by PPAR gamma activators. Biochem Biophys Res Commun 2003;300:472-476.
41. Zeugmann S, Quante A, Heuser I, Schwarzer R, Anghelescu I. Inflammatory biomarkers in 70 depressed inpatients with and without the metabolic syndrome. I Clin Psychiatry 2010;71: 1007-1016.

42. Weber-Hamann B, Kratzsch J, Kopf D, Lederbogen F, Gilles $\mathrm{M}$, Heuser I, et al. Resistin and adiponectin in major depression: the association with free cortisol and effects of antidepressant treatment. J Psychiatr Res 2007;41:344-350.

43. Brunetti L, Orlando G, Recinella L, Michelotto B, Ferrante C, Vacca M. Resistin, but not adiponectin, inhibits dopamine and norepinephrine release in the hypothalamus. Eur J Pharmacol 2004;493:41-44.

44. Yumru M, Gergerlioğlu HS, Savaş HA, Başaralı K, Kalenderoğlu A, Büyükbaş S. Serum resistin levels and metabolic changes in bipolar disorder. J Mood Disord 2012;2:47-50.

45. Dostalova I, Kunesova M, Duskova J, Papezova H, Nedvidkova J. Adipose tissue resistin levels in patients with anorexia nervosa. Nutrition 2006;22:977-983.

46. Rodrigues DH, Rocha NP, Sousa LF, Barbosa IG, Kummer A, Teixeira AL. Changes in adipokine levels in autism spectrum disorders. Neuropsychobiology 2014;69:6-10.

47. Ari M, Ozturk OH, Bez Y, Arica S, Can Y, Erduran D. Serum adiponectin and resistin levels in patients with obsessive compulsive disorder. J Affect Disord 2012;136:979-982.

48. Balõtšev R, Haring L, Koido K, Leping V, Kriisa K, Zilmer M, et al. Antipsychotic treatment is associated with inflammatory and metabolic biomarkers alterations among first-episode psychosis patients: a 7-month follow-up study. Early Interv Psychiatry 2019;13:101-109. 